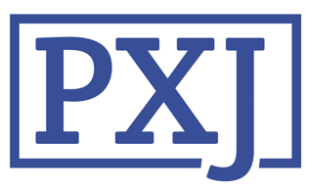

Patient Experience Journal

Volume 5 | Issue 1

Article 14

2018

\title{
What are the most important dimensions of quality for addiction and mental health services from the perspective of its users?
}

Priscilla Liu

Alberta Health Services

Shawn Currie

Alberta Health Services

Jassandre Adamyk-Simpson

Alberta Health Services

Follow this and additional works at: https://pxjournal.org/journal

Part of the Health and Medical Administration Commons, Health Policy Commons, Health Services Administration Commons, and the Health Services Research Commons

\section{Recommended Citation}

Liu P, Currie S, Adamyk-Simpson J. What are the most important dimensions of quality for addiction and mental health services from the perspective of its users?. Patient Experience Journal. 2018; 5(1):106-114. doi: $10.35680 / 2372-0247.1244$.

This Research is brought to you for free and open access by Patient Experience Journal. It has been accepted for inclusion in Patient Experience Journal by an authorized editor of Patient Experience Journal. 


\section{What are the most important dimensions of quality for addiction and mental health services from the perspective of its users?}

\section{Cover Page Footnote}

Acknowledgements This project was funded by a grant from the Canadian Health Services Research Foundation (renamed the Canadian Foundation for Healthcare Improvement). 


\title{
What are the most important dimensions of quality for addiction and mental health services from the perspective of its users? \\ Priscilla Liu, Alberta Health Services, priscilla.liu@albertahealthservices.ca Shawn Currie, Alberta HealthServices, shawn.currie@albertabealthservices.ca Jassandre Adamyk-Simpson, Alberta Health Services, Jassandre.Adamyksimpson@albertahealthservices.ca
}

\begin{abstract}
There is a need to better engage service users in improving their experience with the care received in Addiction and Mental Health (A\&MH). Dimensions of patient experience that are most salient to A\&MH service users still remain to be properly defined from the patient perspective. This research focuses on identifying key domains of service experience important to patients of Addiction and Mental Health using patient focus groups. In addition, through a patient and family advisory committee, patients were also engaged as co-partners of the research team. The patient advisors had a major role in overseeing the research project, assisting with the thematic analysis and identifying the service domains. A total of 48 individuals $(60 \%$ female; mean age $=45$ years $)$ with lived experience using A\&MH services participated in the focus groups. The major themes that emerged from the focus groups led to the identification of seven dimensions of service quality: 1) access, 2) humanity of care, 3) skill and quality of staff, 4) patient engagement, 5) internal and external program communication, 6) individualized treatment and 7) continuity of care. We found that these domains were similar across all service settings including addictions. Patient advisors provided a unique "insider" perspective on the data. Identifying common aspects of service is the first phase of this study. These findings will form the framework for the development of a patient experience survey for Addiction and Mental Health.
\end{abstract}

\section{Keywords}

Patient experience, patient engagement, quality of care, qualitative research, patient centered care

\section{Introduction}

The need to engage patients and improve their experience with the services they receive in Addiction and Mental Health (A\&MH) is well recognized. Historically, individuals with addiction and mental health issues have assumed a very passive role in decisions about their care. Even more rare is the inclusion of A\&MH service users in the planning, delivery and evaluation of services.

Traditional methods of service evaluation and quality improvement are predominantly led by health care professionals. Very few approaches take a "consumer oriented and consumer directed" approach for evaluating mental health services. ${ }^{1}$

The consistent measurement and reporting of patient experience is one way to ensure that services incorporate the perspective of its users in any quality improvement initiatives. ${ }^{2}$ For many health organizations across North America, the H-CAPHS (Hospital-Consumer Assessment of Healthcare Providers and Systems) has emerged as the gold standard instrument to measure patient experience in facility-based acute care services. ${ }^{3}$ The United Kingdom developed a similar hospital patient experience measure based on extensive consultation with health service users. ${ }^{4}$ The mental health care system is lagging behind other health service sectors in the development and adoption of patient experience measures. Although several brief surveys have been developed to assess specific types of A\&MH programs, a single comprehensive instrument with applicability to all service settings is not yet available. More importantly, there has been no extensive involvement of patients to determine the most salient, actionable dimensions of patient's experience in care to develop a measurement tool.

A review of the literature identified that most studies described generating domains of patient experience from time-limited discussions with service users, clinical impressions of staff and consultations with health professionals. ${ }^{5,6,7,8} \mathrm{~A}$ small number of studies described generating domains of care exclusively from the patient perspective 9, 10,11 and only one study involved service users as partners or "consumer researchers" in identifying important service domains and developing experience measures. ${ }^{1(\mathrm{p} 199)}$

As part of a long-term patient engagement strategy, the present project sought to identify the critical domains of patient experience for people who use A\&MH services. The scope of this work included both inpatient and outpatient programs, as well as programs that focus on 
treating addictions. Focus groups were the primary avenue to obtain patient input on the essential dimensions of a high-quality service. In the spirit of the Patient and FamilyCentred Care (PFCC) model, we recruited A\&MH patients to oversee the project. The PFCC model is "grounded in establishing mutually beneficial partnerships". ${ }^{12}$ PFCC means "working in partnership with patients and families and encouraging patients and families to actively participate with members of their health care team in all aspects of health care". ${ }^{12}$ Our project also incorporated the $\mathrm{IAP}^{2}$ Spectrum of Public Participation model which outlines a spectrum of engagement components and techniques. ${ }^{13}$ In the present project, patients were established early in project development as key decision makers in a manner consistent with the involving, collaborating, and empowering components of the IAP2 model. To this end, a patient and family advisory committee was assembled and had the responsibility to provide consumer input into all aspects of the research process and serve as the primary decision-making body for the project.

\section{Methods}

\section{Patient and Family Advisory Committee}

The patient and family advisory committee was made up of an experienced group of individuals with lived experience in using A\&MH services along with frontline service providers and researchers. At least half of the advisory members were made up of patient and family advisors to ensure that patients were well represented and had a strong voice. The selection criteria for patient and family members were: (1) in the past 12 months the individual was a patient (or the first degree relative of a patient) in an inpatient, outpatient or community A\&MH program; (2) psychiatrically stable and possesses good coping skills and strategies for dealing with stress; (3) previous experience as a volunteer or advocate, and; (4) able to provide general feedback regarding A\&MH services based on their own experiences.

All patient and family advisors participated in a screening interview prior to becoming a member. The interview panel included an experienced clinician (SC). If any concerns were raised about the prospective advisor's psychiatric stability, the individual was advised to focus on their treatment rather than take on a role that could exacerbate their symptoms. They were also invited to participate in the focus groups as an alternative.

Participation as an advisor was voluntary; patients and family members could withdraw from the committee at any time.

Because advisory committee members had little experience in quality improvement or research methods, training, orientation and educational sessions were provided to ensure they had working knowledge in patient engagement concepts, survey design and qualitative analysis.
The patient advisors were consulted on formulating the interview guide for the focus groups and assisted in analyzing the anonymous data

\section{Study Sample}

A purposeful and convenience sample was used to recruit A\&MH users for focus groups. Study participants were recruited using posters and word of mouth referrals through service settings in the Calgary area. To be included, participants had to be 18 years or older, and within the past year have experience as an inpatient on a psychiatry unit or attended at least five outpatient appointments with an A\&MH program. Ethical approval was granted by the University of Calgary-Alberta Health Services Conjoint Research Ethics Board and informed consent was obtained before each focus group.

\section{Focus Groups}

The focus groups were conducted in two rounds. The first round used open-ended questions to identify service dimensions that contribute to patients having a positive or negative experience in using the service. The working definition for patient experience was drawn from the Beryl Institute characterization of patient experience as "the sum of all interactions, shaped by an organization's culture, that influence patient perceptions across the continuum of care." 2 (p29) The core focus group questions were modeled after the interview questions employed by Malins et al. (2006) ${ }^{1(\mathrm{p} 198)}$ in their consultations with mental health service users. The core questions were: "Describe a recent positive and negative experience you had with a service (what made it a positive/negative experience?)" and "What are the important aspects of an addiction or mental health service for users?"

Facilitators then used non-leading questions and prompts to elicit further detail from participants. ${ }^{14} \mathrm{~A}$ second round of focus groups was conducted to construct and validate items to assess patient experience within each of the themes. Based on feedback from participants, items were refined to ensure the content captured the experience of A\&MH service users. Focus groups lasted between 60 and 90 minutes and were digitally recorded and transcribed verbatim. The groups were facilitated by two members of the research team. 


\section{Thematic Analysis}

Using established methods, a thematic analysis was completed through a number of stages, starting with reading through the transcripts followed by sorting, reorganizing, and rearranging concepts and ideas into the broad themes and sub-themes. ${ }^{14} \mathrm{~A}$ small working group composed of patient advisors and researchers was formed to complete the qualitative analysis. Patient advisors and researchers collaboratively generated the coding framework. Two coders were assigned to each focus group transcript. A patient advisor was the first coder and a research team member acted as the second coder. There was good consistency among the coders overall and any discrepancies were resolved in the small working group. The extraction of themes occurred until the point of saturation, when the views brought forward were the same as previous views. ${ }^{15}$ The final themes were presented and reviewed by the larger advisory committee.

\section{Results}

\section{Participant Demographics}

In total, 11 focus groups were completed with a total of 48 participants who ranged in age from 20 to 71 years old (Table 1). A representative cross-section of patients with direct experience using inpatient, outpatient, and not-forprofit community agencies was recruited. The sample included a mix of males and females, individuals with both severe and persistent mental illness and episodic disorders, individuals with substance related disorders, and individuals with experience in outpatient or community programs. A range of mental disorders was represented among participants with the most common being mood disorders, anxiety disorders, and addictive disorders. All participants were current users of A\&MH services with three-quarters having prior experience on an inpatient unit; many participants had experience with multiple service settings. Most participants (65\%) were presently taking medication for their illness.

\section{Focus Group Results}

Table 2 shows the domains of patient experience that focus group participants deemed as being important when using A\&MH services. The left hand column shows the high level themes based on the feedback participants provided on what contributes to a positive or negative experience as a user of services. The right hand column of Table 2 shows the subthemes that emerged within the broader domains of care. The subthemes represented the specific program actions, activities, or staff behaviours that characterize the broader theme. For example, a subtheme of "Access" was the individual's ability to receive services in geographic locations that were easily accessible. A total of seven distinct themes emerged: 1) access, 2) humanity of care, 3) skill and quality of staff, 4) engagement, 5) internal and external program communication, 6 ) individualized treatment, and 7) continuity of care. We found that these themes were consistent across all services settings including addictions.

\section{Access}

Participants discussed access to services as being a very important aspect to their experience. Access included various time points of their involvement with a service or program - from the initial referral, to intake, to entry into a program and while they were in the program. It was noted that ease of entry or acceptance into a program is important. Specifically, many participants shared the difficulties they experienced with getting accepted into a program. Some reported having to wait until their condition worsened before they were able to get the help they needed. One participant noted:

I needed to be hospitalized and my doctor drove me to the hospital and that's why I got in...I wasn't eating, I wasn't sleeping, I was pretty well going downhill...the [inpatient unit] is there and it does a good job for a lot of people if they let you in and I think that's the hurdle.

Participants also mentioned the importance of having an efficient intake process that places patients in the most appropriate service easily and quickly. In addition to long wait times with many services, participants identified other access concerns once enrolled in a program notably: seeing their therapist as often as needed; having to 'retell their story' (history of illness, services accessed, treatment needs, etc.) to each new service, and; being informed that another service is actually better suited to their needs and they need to be re-referred. Some participants stated:

Access to some services can take a long time and I had to go into debt to go to private clinics to get some of the help that I needed because I couldn't get in [the program] because there was a 2 year wait list.

Ease of access to service when we need it and when we're able to access the service.

It took my counsellor a few days to get back to me...I called [my counsellor] again... and [my counsellor] never phoned back and weeks went by...I felt like I was putting more effort into meeting with [my counsellor] than the other way around. They didn't get back to me. 
Table 1. Focus group demographics $(n=48)$

\begin{tabular}{lc}
\hline Demographics & $\mathbf{N}(\mathbf{\%})$ \\
\hline Sex & \\
Male & $19(40 \%)$ \\
Female & $29(60 \%)$ \\
Mean age (years) & 44.7 \\
Marital Status & \\
Single & $30(63 \%)$ \\
Married/in a relationship & $8(17 \%)$ \\
Divorced/separated & $10(21 \%)$ \\
Employment Status & \\
Employed (full or part-time) & $11(23 \%)$ \\
Unemployed & $13(27 \%)$ \\
Using social assistance & $23(48 \%)$ \\
Education & \\
Less than high school & $9(19 \%)$ \\
High school & $18(38 \%)$ \\
College/university or higher & $21(44 \%)$ \\
On Medication & $31(65 \%)$ \\
Primary Diagnosis & \\
Depression & $24(50 \%)$ \\
Anxiety disorder & $13(27 \%)$ \\
Bipolar disorder & $7(15 \%)$ \\
Substance abuse and addictive disorders & $6(13 \%)$ \\
Personality disorder & $6(13 \%)$ \\
Schizophrenia & $5(10 \%)$ \\
Other & $10(21 \%)$ \\
Inpatice User Experience & \\
Outpatient & $36(75 \%)$ \\
Emergency/Crisis services & $31(65 \%)$ \\
Addiction services & $22(46 \%)$ \\
\hline & $17(35 \%)$ \\
\hline
\end{tabular}

Once accepted into a service, patients noted the need to have good availability of appointments and be able to see their health provider as often as needed. Finally, participants identified the need to have programs and services in locations that are easily accessible. One participant stated:

I don't live in [city], no programs offered in the rural areas.

\section{Humanity of Care}

In all the focus groups, participants identified the importance of "being respected and treated like a human being." This included respecting patient choices and rights and the need to feel heard, validated and understood.. Participants frequently stated that they desired to have a voice including being able to raise a complaint or issue and have it addressed or taken seriously. They also desired to be treated with dignity and equally without judgement or being labelled or stigmatized based on their illness. Finally, participants highlighted the importance of having their privacy and confidentiality protected and the need to feel safe to openly and honestly share with their provider. Examples of what participants described relating to this theme included: 
Table 2. Domains of patient experience

\begin{tabular}{|c|c|}
\hline Theme & Sub-Themes \\
\hline Access & $\begin{array}{ll}\text { - } & \text { Wait times } \\
\text { - } & \text { Intake process } \\
\text { - } & \text { Location of facility } \\
\text { - } & \text { Once in service, access to staff }\end{array}$ \\
\hline Humanity of Care & $\begin{array}{l}\text { - } \text { Being treated like a human being } \\
\text { - } \text { Respecting patient rights and choices } \\
\text { - } \text { Having a voice } \\
\text { - } \\
\text { Protection of privacy and confidentiality }\end{array}$ \\
\hline Skill and Quality of Staff & $\begin{array}{l}\text { - Interpersonal skills and qualities (empathetic, compassionate, genuine) } \\
\text { - Technical skills of staff (professional, knowledgeable, competent) }\end{array}$ \\
\hline Patient Engagement & $\begin{array}{l}\text { - Involving patients in decision making regarding their care } \\
\text { - Involving families or loved ones in decisions about treatment and care }\end{array}$ \\
\hline Communication & $\begin{array}{l}\text { - Information sharing with patient } \\
\text { - Information sharing between health care providers within a program and } \\
\text { between programs and services }\end{array}$ \\
\hline $\begin{array}{l}\text { Individualized } \\
\text { Treatment }\end{array}$ & $\begin{array}{l}\text { - } \text { Receiving the appropriate screening and assessment } \\
\text { - Treatment effectiveness leading to reduced symptoms or improved } \\
\text { - } \text { outcomes } \\
\text { - } \text { Individualized and comprehensive treatment plan } \\
\text { - } \quad \text { Having treatment options } \\
\text { - } \quad \text { Consistency of care }\end{array}$ \\
\hline Continuity of Care & $\begin{array}{ll}\text { - } & \text { Discharge planning } \\
\text { - } & \text { Connecting to community supports/community transition } \\
\text { - } & \text { Follow-up }\end{array}$ \\
\hline
\end{tabular}

[Staff] didn't treat you like you are an alcoholic/drug user, they treat you like a human being.

We all have feelings and emotions and rights and our rights have been stripped from us and labeled and we don't have a voice....we should be treated with respect...we're terrified and we're afraid to open up.

Realizing the importance of the person rather than a number and being willing to listen to what they're really having problems with rather than what fits into this certain criteria [for diagnosis].

They take the time, they are attentive, give eye contact, make you feel like you are important.
Basically how they treat me. Are they taking me seriously? Are they treating me with compassion and empathy and understanding?

Our privacy and confidentiality are removed [in the inpatient unit]. If there's something [health care staff] thinks [a family member] should know, they just do it. It's just assumed that if someone is married to you, [the person] is a safe person to tell and that's not a safe assumption.

\section{Skill and Quality of Staff}

Another theme that emerged from the focus groups was related to skill and quality of staff. Participants listed a set of skills and qualities possessed by staff that were 
important to them. These included having good communication skills; being able to listen and empathize with the patient. They also desired a health provider that expresses hope and gives positive messages.

Examples of what participants mentioned included:

I think the professionals that you work with, having the knowledge, skill, diligence, empathy, and being able to demonstrate those things when you are engaged with them. I think that's very important.

[Staff] who are supportive and encouraging, and they don't talk down to us. They are very positive, happy, and has a focus on wellness.

Furthermore, participants noted the importance of staff to be genuine and supportive and expressing care and compassion. They frequently mentioned the significance of having "a connection" with their provider who will respond to their needs.

Two participants noted:

If people don't have heart and compassion they should not go into this profession.

Someone who has empathy, and someone you can connect with.

Lastly, participants felt it was important for staff to have strong technical skills and be knowledgeable and competent in their field. As one participant stated:

I like that [staff] don't just give you what you need to hear but they give you feedback and how you can do things that are different and productive, different ways of thinking.

\section{Patient Engagement}

Engaging patients and families was identified as being an essential aspect to their experience. Patients would like to work with staff in making decisions about what kind of treatment and care is best for them. Families of patients are also another group that was identified as important to engage. However, it was expressed that while not all patients may necessarily want to have family involvement, including family members should be offered as much as desired. Two participants described what they felt would be the best way to engage patients and family members:

The ideal service is that you are consulted and you make a decision together.

What I look for when I'm acquiring a service is someone who can be consistent and also that they will incorporate my insight and knowledge of my illness into the treatment practices. And also that they will acknowledge my family and have their input as well.

\section{Communication}

This theme is related to information sharing with patients and between health care providers. Participants noted that they like to feel informed of what is going on while they are in care and that they prefer information to be shared in a clear and consistent way. One participant shared:

Sometimes you're left alone for a long time [in the emergency department], and not even being communicated to about what's going on, what's happening. You need to have someone there to say, “OK, we're going to see this many people, we'll come back to you. And in the meantime, this is what's going to happen".

The sharing of information should also be extended to partners and family members or caregivers according to the patient's wishes. Specifically, basic information that they identified as important included names along with roles and responsibilities of staff when introducing themselves. Participants also mentioned being given enough information and education about their condition or issue as stated by one participant:

I think it's important so that you know what you're dealing with...but with the education and awareness, there were things you just didn't know. So the education is key.

Another part to the theme of communication involves communication between health care providers within a program and between programs and services. Participants highlighted that often times information sharing between health providers is not consistent or timely. One participated mentioned:

I feel disconnected with my key counsellor and then have to summarize to them my two weeks, what I've experienced, what I've shared and what's going on [from group counselling].

\section{Individualized Treatment}

Under this theme, participants discussed treatment that is tailored to the individual's needs and goals for reduced symptoms and improved quality of life as being important. Participants wanted a comprehensive approach to their treatment that addressed other needs such as social isolation, physical health, life skills and basic needs in addition to mental health. Being given treatment options and individualizing their treatment plan with specific goals was preferred along with receiving timely access to appropriate screening and assessments. Examples of what participants brought forward included:

It has to be tailored, because everyone's [illness/issue] involves different things. 
Sometimes, it's assumed that because of your diagnosis, you should fit in a particular hole and a particular treatment will work. But not everyone is the same, and they still try to shove you in that hole whether you fit or not.

I think it would be important to ask questions about how to put the big picture together, and how that's coming together for each and every person. So your housing, your financial situation, work, education if that's applicable, as well as your psychiatric treatment and how well that's actually going.

In my situation, they should have checked back to see if taking [medication] as a child had any connection with the diagnosis I've had, and run tests, which they did not. So they jump to conclusions because of the way I communicate with them, but in logical tests I'm fine. So to my knowledge, they didn't do the proper tests.

Another sub-theme was having peer support or the opportunity to connect with other peers as being significant to their recovery as noted by one participant:

I've noticed that the little bit of activity that we've done, our group got tighter and broke down boundaries and broke down that wall for me personally. We can show each other the strong side and vulnerable side and help out the healing process. Cause I know for me if it wasn't for the small activities, group sessions, etc and the interactions with [my peers], I wouldn't have felt secure enough to walk out of here and say ok I am not going to relapse.

Finally, participants feel that it is important to be able to receive consistency of care from the same provider or service for as long as needed.

\section{Continuity of Care and Community Transition}

Patients reported that having continuity of care to help with transitioning into community after being discharged as very important to their long term recovery. This includes having a discharge plan with appropriate community referrals and resources in place before leaving a service and discussing the discharge plan in advance. Two participants stated:

It wasn't just having a [discharge] plan, but knowing what it was.

If I am discharged, I'm worried about not having access to or knowing what programs are coming up.

In addition, having some kind of follow-up to help patients stay well or having a point of check-in is desired as one participant shared:
Staff reaching out to you, even after being discharged from a program. On-going check-in.

Finally, continuity of care should include supports for patients that will address basic needs, continue to build life skills and independent living skills and offer social and recreational activities. For example, one participant noted:

Being involved in community... building social relationships, a chance to connect with people.

\section{Discussion}

The goal of the first phase of our research project was to utilize patient input to identify the dimensions of A\&MH service that are most important to a positive experience of users. This was achieved through extensive consultation with service users via focus groups. Higher level themes and subthemes of patient experience were extracted from these consultations. The most innovative aspect of the project was the role of patients in the analysis and reporting process. Patient advisors were an integral part of identifying themes of service experience from focus group transcripts. In addition to being an excellent application of patient engagement, using advisors to extract themes had several practical advantages. Foremost, patient advisors provided a unique, 'insider' perspective on the data. As users of A\&MH services themselves, they could more easily interpret comments from other patients, and extract the most relevant theme. Patient advisors were also highly skilled at re-framing subthemes as actions that will aid in phase two of our study in forming the basis for a survey question. As noted by Balogh and colleagues (1995) ${ }^{16}$ the concepts of good or poor service quality are hard to define in the context of A\&MH services but are easy for patients themselves to recognize when they have direct experience. Patient advisors can also help researchers understand the feedback of fellow service users on what contributes to a positive or negative experience.

Service users identified seven salient dimensions of service: 1) access, 2) humanity of care, 3) skill and quality of staff, 4) engagement, 5) communication, 6) individualized treatment, and 7) continuity of care. Interestingly, these themes were similar for both addiction and mental health patients. There is overlap between these dimensions and those identified in the study by Malins et al. (2006) 1(p199) which employed a similar methodology. Notably, the desire for services to promote patient involvement in care, communication skills of staff, humanity of care, and the importance of a positive staff-patient relationship. There is also similarity between these themes and the dimensions of care covered by more general patient experience surveys for medical services. For example, $\mathrm{H}_{-} \mathrm{CAHPS}^{3}$ covers staff communication, responsiveness of staff, and quality of discharge planning. 
Within each of the broader dimensions of care, subthemes emerged that were unique to A\&MH service users. The emergence of these subthemes provides a strong rationale for the use of a patient experience measure that is tailored to the population of A\&MH service users. For example, within the dimension of 'Humanity of Care' emerged the importance of respect and avoiding stigmatizing actions. Under the dimension of 'Individualized Treatment' emerged the subtheme of providing comprehensive, biopsychosocial treatment that addresses more than just symptom reduction.

\section{Limitations}

One of the main limitations of this work is the exclusive focus on adult service users. Approximately $30 \%$ of patients receiving publicly funded A\&MH services in our health region are under 18 years of age. Because of funding and time constraints, we did not incorporate advisors representing the child and adolescent mental health services into the project. However, shortly after the current project was underway, the senior leadership for child and adolescent mental health services established a parallel patient advisory committee composed of parents, clinicians, and management with a broad mandate to provide consumer input into planning and quality improvement initiatives.

Another limitation is the exclusion of psychiatrically unstable individuals in the patient and family advisory committee. However, their views were still captured in the focus groups and represented in the themes that emerged.

\section{Next Steps}

The themes and subthemes that emerged from the focus groups will form the basis for a draft patient experience survey. The survey will undergo cognitive and comprehensive psychometric testing. A large sample of A\&MH service users, representing a range of treatment settings (inpatient, outpatient, addictions), will complete the survey. Testing will establish the survey's underlying scale structure, test-retest reliability, concurrent and predictive validity, and internal consistency. The results will be presented in a subsequent paper.

Modelling the principles of Patient and Family-Centered Care, the project advisory committee has been established as a permanent patient experience advisory council for our health region's adult A\&MH services. New members have been recruited with an expected term of two years and at least half of the advisory is made up of patient or family advisors. The advisory committee is co-chaired by a patient or family member. The main function of the council is to review the results from the new survey and provide input to programs on quality improvement strategies to address items with low scores.
Representatives from the programs under review (typically the manager and frontline staff) attend the meeting to participate in the discussion. The intention is to provide the program providers with concrete suggestions for enhancing the patient experience. In addition to reviewing survey results, the council is often asked to provide input on planning and service enhancements.

\section{Lessons Learned}

There were many lessons learned through the process of establishing a patient and family advisory committee and engaging patients in improving services. Both the process and tangible outcomes realized were extremely rewarding to our organization and we highly recommend A\&MH services in other jurisdictions to undertake similar initiatives to engage patients in quality improvement work. For other health care organizations interested in undertaking similar work, the following are some of the key learnings:

1) The process described in this report requires a considerable investment of time and resources to engage service users in a meaningful way.

Additional time and effort was required to recruit patients and family members, provide orientation and training, and build mutual trust and respect in the relationship.

2) Offering orientation, training, and support to patient and family advisors is important for them to be effective in their role. Orientation helped patient advisors understand their role and the value that they bring with their unique perspective as service users. Educational sessions on research and quality improvement methods also provided the patient advisors with sufficient knowledge on these topics and the confidence to be able to contribute to the discussions.

3) The balance in the number of patients/family members and health care providers is essential to ensure that patients and family members have a strong voice and are equal partners in improving care. A true patient-led quality improvement team should be composed of at least fifty percent service users.

4) Finally, there is need for consideration of sustainability planning that includes identifying processes, engaging staff, and shifting the organizational culture to move towards including the patient perspective in the design, delivery, and evaluation of A\&MH services.

\section{References}

1. Malins G, Oades L, Viney L, Aspden S. What's in a service? Consumers' views of Australian mental health services. Psychiatric Rehabilitation Journal. 2006; 29: 197 204. 
2. LaVela SL, Gallan AS. Evaluation and measurement of patient experience. Patient Experience Journal. 2014; 1: 28-36.

3. HCAHPS Survey: Survey Instruments. HCAHPS Hospital Consumer Assessment of Healthcare Providers and Systems Website. http://www.hcahpsonline.org/surveyinstrument.aspx. Assessed July 2017.

4. Davies E, Cleary P D. Hearing the patient's voice? Factors affecting the use of patient survey data in quality improvement. Quality \& Safety in Health Care, 2005;14(6), 428-432.

5. Barker DA, Orrell MW. The Psychiatric Care Satisfaction Questionnaire: A reliability and validity study. Social Psychiatry and Psychiatric Epidemiology. 1999; 34: 111-116.

6. Brunero S, Lamont S, Fairbrother G. Using and understanding consumer satisfaction to effect an improvement in mental health service delivery. Journal of Psychiatric and Mental Health Nursing. 2009; 16: 272278.

7. Kolb SJ, Race KEH, Seibert JH. Psychometric evaluation of an inpatient psychiatric care consumer satisfaction survey. The Journal of Behavioral Health Services \& Research. 2000; 27(1): 75-86.

8. Lelliott P, Beevor A, Hogman G, Hyslop J, Lathlean J, Ward M. Carer's and Users' Expectations of Services - User version (CUES-U): a new instrument to measure the experience of users of mental health services. The British Journal of Psychiatry. 2001; 179: 6772.

9. Gigantesco A, Morosini P, Bazzoni A. Quality of psychiatric care: validation of an instrument for measuring inpatient opinion. International Journal for Quality in Health Care. 2003; 15(1): 73-78.

10. Langle G, Baum W, Wollinger A, et al. Indicators of quality of inpatient psychiatric treatment: the patients' view. International Journal for Quality of Health Care. 2003; 15(3): 213-22.

11. Meehan T, Bergen H, Stedman T. Monitoring consumer satisfaction with inpatient service delivery: the inpatient evaluation of service questionnaire. Australian and New Zealand Journal of Psychiatry. 2002; 36: 807-811.

12. What is PFCC? Institute for Patient- and FamilyCentered Care website. http://www.ipfcc.org/about/pfcc.html. Accessed July 2017.

13. IAP2's Public Participation Spectrum. International Association for Public Participation Website.https://c.ymcdn.com/sites/www.iap2.org/re source/resmgr/foundations_course/IAP2_P2_Spectr um_FINAL.pdf. Published 2014. Accessed July 2017.
14. Padget DK. Qualitative Methods in Social Work: Challenges and Rewards. Thousand Oaks, CA: Sage Publications; 1998.

15. Krause N. A comprehensive strategy for developing closed-ended survey items for use in studies of older adults. The Journals of Gerontology. 2002; 57(S): 263-274.

16. Balogh R, Simpson A, Bond S. Involving clients in clinical audits of mental health services. International Journal for Quality in Health Care.1995; 7: 343-353. 